\title{
REVISTA
}

Revista Educación

ISSN: 0379-7082

ISSN: 2215-2644

revedu@gmail.com

Universidad de Costa Rica

Costa Rica

\section{Evaluación del currículo ofertado por la Universidad Estatal Amazónica para la carrera de Ingeniería en Turismo}

Coromoto Marín, Haideé; Soria Velasco, Román Eduardo; Bravo Medina, Carlos Alfredo; Reyes Vargas, María Victoria; Gamboa Ríos, María Germania; Manjarrez Fuentes, Nelly Narcisa; del Corral Villaroel, Víctor Hugo

Evaluación del currículo ofertado por la Universidad Estatal Amazónica para la carrera de Ingeniería en Turismo

Revista Educación, vol. 42, núm. 2, 2018

Universidad de Costa Rica, Costa Rica

Disponible en: http://www.redalyc.org/articulo.oa?id=44055139022

DOI: https://doi.org/10.15517/revedu.v42i2.26513

Esta obra está bajo una Licencia Creative Commons Atribución-NoComercial-SinDerivar 3.0 Internacional. 


\section{Evaluación del currículo ofertado por la Universidad Estatal Amazónica para la carrera de Ingeniería en Turismo}

\section{Curriculum Evaluation of Major in Tourism Engineering Offered by The Amazon State University}

Haideé Coromoto Marin [1]

Universidad Estatal Amazónica, Ecuador

DOI: https://doi.org/10.15517/revedu.v42i2.26513

hmarin@uea.edu.ec

iD http://orcid.org/0000-0001-7276-7401

Román Eduardo Soria Velasco [2]

Universidad Estatal Amazónica, Ecuador

rsoria@uea.edu.ec

Carlos Alfredo Bravo Medina [3]

Universidad Estatal Amazónica, Ecuador

cbravo@uea.edu.ec

\section{Notas DE AUTOR}

[1] Licenciada en Docencia Agropecuaria, Doctora en Planificación, Dirección y Entorno Económico Social de la Empresa por la Universidad de Córdoba-España, más de 20 años como docente. Tutora de tesis, asistencia a congresos y trabajos publicados en revistas científicas arbitrada. Experiencia como sub-directora Académica y de Investigación. Directora Académica de la Universidad Estatal Amazónica. Responsable de Elaborar Proyectos de diseños curriculares de Pregrado y Posgrado. Coordinadora de Proyectos de Investigación. Publicaciones: Los retos de la Educación en el contexto Universitario, Caracterización biofísica y social de la sub cuenca alta del río de San Pedro, Estado Miranda; "Income Distribution of Protected Crop Farms in Andalucia”; An integrated model to study the structural and financial sustainability of agricultural enterprises”.

[2] Ing. Alimentos, Magister Ciencia y Tecnología de la Leche. Docente principal Universidad Técnica Estatal de Quevedo desde febrero 1982. Decano Facultad de Ciencias Pecuarias. Miembro Comisión Planificación y Evaluación. Director de Planificación y Evaluación. Asesor Planificación y Evaluación y vicerrector Administrativo Universidad Estatal Amazónica. Evaluador Externo procesos Acreditación Universidades Ecuatorianas. Equipo evaluación Consejo Nacional Educación Superior. Tutor de tesis, asistencia a congresos y trabajos publicados en revistas científicas arbitrada. Comisión de Diseños Curriculares. Publicaciones: Queserías rurales en Los Andes: La experiencia de Salinas- Ecuador. Tecnología de cereales. Manual de estudio para Ingenieros en industrias pecuarias.

[3] Ing. Agrónomo, Doctor en Ingeniería Ambiental por la Universidad de Córdoba-España, con más de 20 años de experiencia docente investigador. Coordinador de Proyectos Multidisciplinarios. Ha participado en congresos, cursos y conferencias como ponente en México, Argentina, Ecuador, Bélgica, Brasil, entre otros. Comisión de diseños curriculares. Publicaciones: Selección del diseño de muestreo en parcelas experimentales a partir del estudio de variabilidad espacial de los suelos., analysis of soil chemical properties and pea yield in a reduce tillage experiment in southern Spain. In: IV Meeting of Precision Agriculture, Berlín -Alemania; term tillage effect on the distribution of phosphorus fractions in a Vertisol of Southern Spain. European Journal of Agronomy.; Socio-Environmental Characterization of Agricultural Production Units in the Ecuadorian Amazon Region.

[4] Ingeniera en Ecoturismo, Doctora en Ciencias Económica por la Universidad de las Villas-Cuba. Decana de la Facultad de Ciencias de la Vida, con más de 10 años de experiencia profesional como docente investigadora. Coordinadora de Proyectos Ha participado en congresos, cursos y conferencias. Publicaciones: Evaluación de territorios para desarrollar el turismo comunitario en la región amazónica del Ecuador. La gestión del turismo comunitario en el marco de la economía popular y solidaria en Ecuador. Propuesta metodológica de evaluación del turismo comunitario, caso de estudio en Arajuno, Pastaza. Tourism strategies for "advantaging" the Amazon rainforest region.

[5] Ingeniera en Ecoturismo, Magister en Gerencia de proyectos. Docente con más de cinco año de experiencia. Coordinadora de la carrera de Turismo en la UEA. Coordinadora de proyectos de investigación. Ha participado en congresos y seminarios. Publicaciones: Diagnóstico socio ambiental del Centro de Investigación, Posgrado y Conservación de la Biodiversidad Amazónica (CIPCA) para la planificación de uso público, Turismo comunitario: Análisis situacional en el cantón Tena, provincia de Napo, El CIPCA y su entorno: Conociendo sus potencialidades para el desarrollo turístico Huellas del Sumaco.

[6] Doctora en Ciencias Económicas por la Universidad de la Habana-Cuba. Vicerrectora Académica de la Universidad Estatal Amazónica. Más de 20 años de experiencia profesional como docente en Facultad de Ciencias Empresariales, unidad de estudios a Distancia y Posgrado. Ha participado en congresos, cursos y conferencias como ponente en Universidades de Cuba, Bolivia, Ecuador y Colombia. Comisión de diseños curriculares. Publicaciones: Reflexiones sobre el concepto de capital humano desde la teoría económica, Algunas consideraciones sobre el crecimiento y desarrollo económico, Desde la perspectiva de sostenibilidad, la gestión del turismo comunitario en el marco de la economía popular y solidaria en Ecuador.

[6] Licenciado en Turismo y Hotelería, Magister en Gerencia de Proyectos Ecoturísticos. Profesional con más de cinco años de experiencias en el área docente, asesor de proyectos ecoturísticos. Aspirante a Doctor. Publicaciones: Desarrollo turístico de Pastaza: Diagnóstico estratégico participativo ponderado, Diagnóstico prospectivo del potencial turístico de Pastaza. 


\author{
María Victoria Reyes Vargas [4] \\ Universidad Estatal Amazónica, Ecuador \\ mreyes@uea.edu.ec \\ Maria Germania Gamboa Rios [5] \\ Universidad Estatal Amazónica, Ecuador \\ mgamboa@uea.edu.ec \\ Nelly Narcisa Manjarrez Fuentes [6] \\ Universidad Estatal Amazónica, Ecuador \\ nellynmf@yahoo.es \\ Victor Hugo del Corral Villaroel [6] \\ Universidad Estatal Amazónica, Ecuador \\ vdelcorral@uea.edu.ec
}

Recepción: 27 Enero 2017

Aprobación: 20 Marzo 2018

\title{
Resumen:
}

El currículo, para ser exitoso y de calidad, debe estar siendo evaluado permanentemente, con intención de mejorarlo y lograr así la plena formación del estudiantado. Esta se hace cada vez más importante, debido a que permite generar y obtener logros significativos en el proceso educacional en pro de un perfeccionamiento racional y científicamente válido para enfrentar los desafíos actuales. El objetivo de esta investigación fue realizar la evaluación curricular de la carrera de turismo de la UEA, a través de una metodología con enfoque epistemológico orientado al cambio. Se utilizó el método de la investigación acción participante, que consiste en un proceso sistemático que requiere la participación de todos los actores involucrados en el proceso de enseñanza aprendizaje (internos y externos), quienes tienen el conocimiento más profundo de sus problemas y su realidad. Los resultados de la investigación muestran los hallazgos, fallas, debilidades y aciertos que orientaron la toma de decisión para realizar los ajustes pertinentes y favorecer la calidad de la enseñanza, que es uno de los grandes desafíos de la educación superior del siglo XXI.

Palabras Clave: Evaluación curricular, evaluación interna, evaluación externa, turismo.

\section{ABSTRACT:}

Any curriculum must be continuously evaluated to be successful and high quality in order to improve and achieve complete student formation. In fact, this becomes highly important because it allows generating and obtaining significant achievements in the educational process towards a rational and scientifically valid development to face the current challenges. The aim of this research was to conduct the curriculum evaluation of the Tourism major at Amazon State University (AEU) through a methodology with a change-oriented epistemological approach. A participatory - action research method was used consisting of a systemic process where the participation of all actors involved in the educational process are required, even internal or external ones, since they have the deepest knowledge of their problems and reality. The results of the research show the findings, failures, weaknesses and strengths used as a base to solve them and promote teaching quality which is one of the major educational challenges in the XXI century.

KEYWORDS: curriculum evaluation, internal evaluation, external evaluation, tourism.

\section{INTRODUCCIÓN}

A nivel mundial, el sector educativo, en los últimos años, ha sido sometido a grandes cambios, especialmente el sector universitario, en un intento de acercar más los planes y programas de estudio a la sociedad, para que respondan a las nuevas demandas, en armonía con las tendencias actuales de los modelos curriculares. Por su parte, Marín (2015), en el proyecto de investigación financiado por la SENESCYT titulado: Propuesta metodológica para la actualización curricular de syllabus de las carreras dictadas en la UEA, indica: 
El Sistema Educativo Universitario ecuatoriano no escapa de esa realidad y durante los últimos años ha pasado por una serie de vertiginosos cambios, que intentan dar respuesta a los problemas y necesidades evidenciados en la realidad educativa. Dentro de esta necesidad de mejora, se le ha dado a la Educación Superior una importancia fundamental y casi estratégica, debido al compromiso que tienen las universidades en su papel de formadoras de profesionales en pro del desarrollo del país al considerarse como un elemento dinamizador y multiplicador de cambios que repercuten al mejoramiento de la calidad de la educación en general. (p. 4)

Son diversos los llamados de atención (UNESCO, Proyecto Tunning, Declaración de Bolonia, Informe Delor, etc.) que desde finales del siglo pasado se le vienen haciendo a la educación superior para que cambie sus procesos y presenten propuestas que respondan a las necesidades del nuevo siglo.

Por ello, para garantizar el éxito y la calidad de los programas de estudios, el currículo debe estar siendo evaluado permanentemente con intención de mejorarlo, actualizarlo y adecuarlo de manera oportuna y racional, según la situación educativa y social del contexto actual para lograr la plena formación académica de profesionales en este plan de estudio. Por lo tanto, se hace necesario identificar los aciertos, fallas y debilidades, en el afán de plantear soluciones para resolver los problemas detectados. En este sentido, Roldán (2004), plantea que para que un plan de estudio genere cambios socio culturales y favorezca el desarrollo científico y tecnológico, es necesaria su evaluación.

A través de la evaluación curricular se puede mantener el seguimiento permanente que evite, en condiciones determinadas, que los planes de estudios se conviertan poco a poco en obsoletos. Por lo cual, se hace indispensable valorar lo más objetiva y sistemáticamente posible los logros y deficiencias del plan curricular (Díaz, 2005; Salas, 2016).

En este contexto, la evaluación curricular se puede considerar como un proceso dinámico y complejo que tiene como finalidad informar sobre el progreso, debilidades y fortalezas académicas detectadas en la ejecución de los programas educativos, y buscar alternativas de solución para mejorarlas. Se coincide con Briceño y Chacín (2008), quienes lo conciben como un proyecto social que implica un proceso dialectico, complejo, crítico, reflexivo y participativo para lograr cambios que favorezcan el proceso educativo. Esto implica valorar en profundidad el proceso de enseñanza aprendizaje del estudiantado, las metodologías de enseñanza, los recursos de aprendizaje e instrumentos de evaluación empleados.

La evaluación curricular se hace cada vez más necesaria para enfrentar las nuevas demandas de la sociedad, ya que sus resultados permiten tomar decisiones adecuadas y oportunas para mejorar la calidad y excelencia de los programas educativos (Mata, 2010; Mateo, 2008).

Con base en lo anterior, el objetivo de este trabajo de investigación fue realizar la evaluación curricular del plan de estudio de la carrera de turismo ofertado por la Universidad Estatal Amazónica (UEA), a través del modelo evaluativo propuesto por García et al (2013) con un enfoque epistemológico orientado al cambio.

\section{Metodología}

La investigación se organizó en tres fases: a) planificación del proceso y recopilación de información secundaria y primaria. Las fuentes secundarias, comprendieron una revisión bibliográfica tanto en soporte tradicional como en internet de estudios teóricos prácticos realizados sobre la evaluación curricular, que sirvió de apoyo para establecer las bases teóricas de la investigación. Las fuentes primarias (Tablas 1 y 2 ) fueron, indudablemente, las más sólidas y valiosas, producto de la información aportada por encuestas a los distintos actores involucrados en la investigación (estudiantes, docentes, estudiantado graduado, entes empleadores y autoridades); b) tabulación, análisis e interpretación de los resultados. 
TABLA 1

Población académica encuestada

\begin{tabular}{llll}
\hline Género & Docentes (\%) & Estudiantes (\%) & Graduados/as (5) \\
\hline Mujeres & $20(44)$ & $21(75 \%)$ & $13(65)$ \\
Varones & $25(56)$ & $7(25 \%)$ & $7(35)$ \\
\hline Total & 45 & 28 & 20
\end{tabular}

Nota: Elaboración propia.

TABLA 2

Población empleadora encuestada

\begin{tabular}{ll}
\hline Sector & Entes empleadores (\%) \\
\hline Público & $12(86)$ \\
Privado & $2(14)$ \\
\hline Total & 20
\end{tabular}

Nota: Elaboración propia

Para ello se emplearon, de manera combinada, métodos teóricos (analítico-sintético), empíricos (la observación) y el enfoque epistemológico orientado al cambio y a la toma de decisiones. Se utilizó el método de la investigación acción participante propuesto por Fals Borads, que constituye un proceso sistemático donde se requiere la participación de todos los actores involucrados, quienes conocen a profundidad sus problemas y la realidad (Ortiz y Borjas, 2008).

Considerando las tenencias actuales en cuanto a modelos curriculares que tienen que romper con las posturas epistemológicas tradicionales: positivismo, conductismo, e ir hacia modelos más dinámicos, flexibles y contextualizados, se siguió el modelo evaluativo propuesto por García Sanz et al. (2013), ajustado por el equipo autor al contexto de la UEA. El modelo señala, entre otras cosas, que la evaluación de un programa implica recolección, análisis e interpretación de información fiable que favorezca la toma de decisiones. Este modelo de evaluación, incluye una evaluación interna y otra externa. La evaluación externa se refiere al impacto social que puede tener la labor del estudiantado egresado y la carrera, en la solución de problemas relacionados con su perfil y área de conocimiento. A su vez, la evaluación interna hace énfasis en la organización de los aprendizajes y la estructura curricular que harán posible el logro de los resultados de aprendizaje establecidos en el perfil profesional. El éxito de esta valoración está sujeto a la participación colectiva de todos los actores involucrados en el proceso (sociedad, estudiantes, docentes, entes evaluadores, etc.).

\section{RESULTADOS Y DISCUSIÓN}

\section{Evaluación Interna}

Uno de los términos más debatidos y controversiales en el ámbito educativo es quizás la evaluación curricular, sin embargo, distintos estudios coinciden que el fundamento de esta es detectar inconsistencias y debilidades en su ejecución, a través del análisis crítico de información confiable y relevante, que permita a los entes 
responsables del sector educativo tomar las decisiones pertinentes para alcanzar la excelencia académica (Inciarte, A, 1996; Salas, P. y Salas, M., 2014; Sánchez, M., 2010; Inciarte y Canquiz , 2009).

En este contexto, vale la pena resaltar lo expresado por Fonseca y Pereira (2011) con respecto a la evaluación curricular:

La evaluación posibilita que el proceso educativo sea visto como un ciclo dinámico y sistemático que presupone la existencia de ciertos objetivos que han sido construidos en la etapa de planificación. La esencia de la evaluación se centra en la verificación de la etapa de ejecución o implementación, lo cual permite aplicar las medidas correctivas que se estimen pertinentes. En suma, la evaluación vista como un ejercicio continuo coadyuva al aseguramiento de la efectividad de las acciones en relación con los logros que se pretenden alcanzar. (p. 11)

En el caso de la evaluación curricular interna, su fin último es determinar si el currículo es pertinente, eficiente y eficaz para responder a las demandas tanto de la sociedad en su conjunto como de la institución evaluada (Inciarte y Canquiz, 2001; Pérez, 2007).

Para ello se consultó la opinión de docentes y estudiantes (Tabla 1) sobre la pertinencia de los contenidos, su relación con el perfil de egreso, y estrategias para realizar una actualización de los planes de estudios que responda a las demandas y necesidades de la sociedad, estudiantes, estudiantado graduado y los objetivos educativos de la Universidad. En la Figura 1 (1a), se observa la opinión de estudiantes de los últimos semestres de la carrera en relación con la preparación del personal docente, el 68\% manifestó que el profesorado tiene una buena preparación y solo un $11 \%$ expresó una preparación académica regular. Estos resultados se corresponden con las exigencias de la UEA, ya que para ingresar al escalafón docente se debe tener título de cuarto nivel, es decir, como mínimo el grado de maestría.

En cuanto a las estrategias de enseñanzas y evaluación empleadas por docentes, el $47 \%$ consideró que eran buenas, un $39 \%$ regular y solo un $14 \%$ muy buena (Fig. 1b).
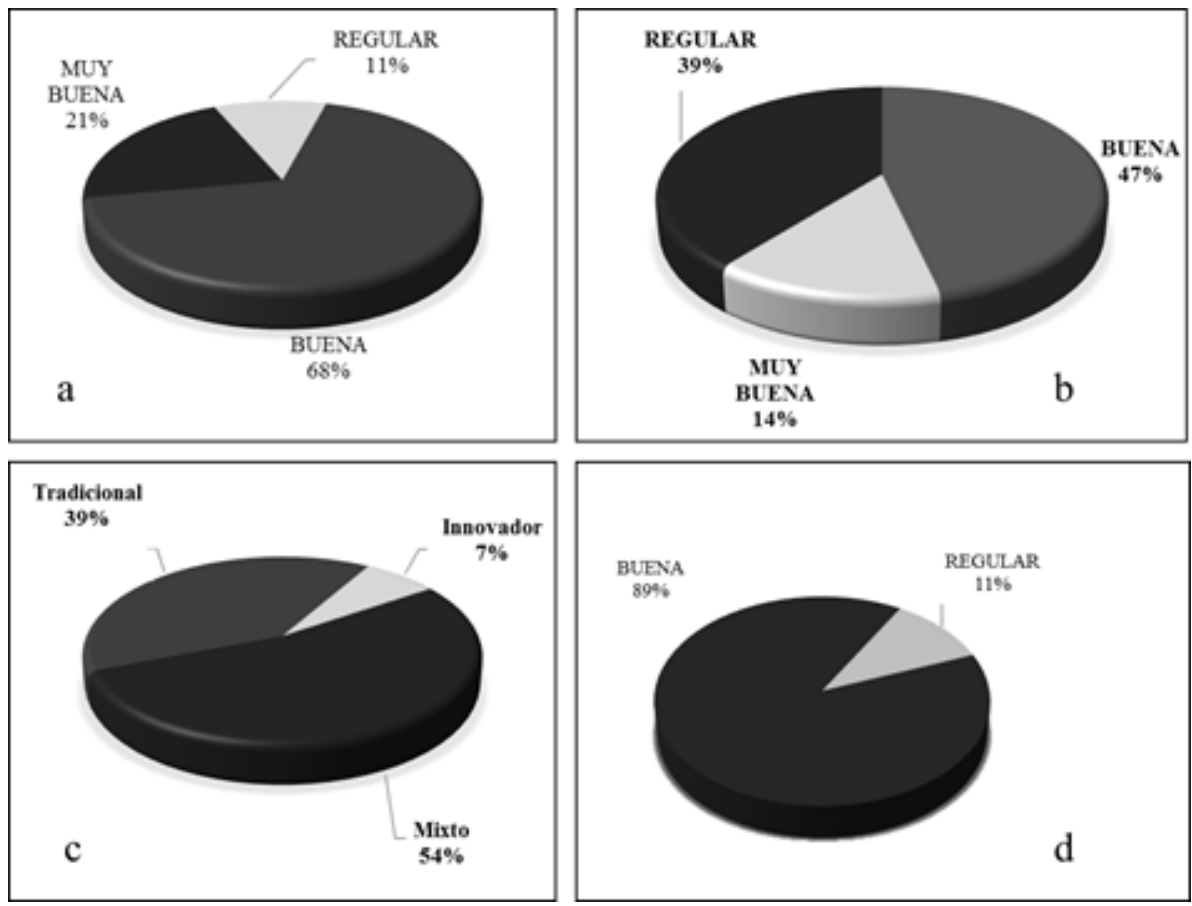

FIGURA 1

1(a) Preparación académica de docentes; 1(b) Estrategias de enseñanza y evaluación; 1 (c)

Categorización de docentes y 1 (d) Pertinencia, actualización y coherencia de los contenidos del sílabo con el perfil del estudiantado egresado (Fuente: Investigación de campo).

Aunque la selección de las estrategias de enseñanza-aprendizaje es responsabilidad exclusiva del personal docente, los resultados indican que un 39\% de estudiantes no está conforme con esas estrategias, algunos 
argumentos esgrimidos manifiestan que no son motivadoras y no generan aprendizajes significativos. En este caso, la metodología de enseñanza-aprendizaje utilizada por docentes debe estar en correspondencia con el Modelo Educativo de la Universidad, basado en el logro de los resultados de aprendizajes desde el paradigma de la complejidad, es decir, requiere un cambio en las relaciones docente-estudiante-sociedad, durante el proceso formativo, de tal manera que les permita a sus estudiantes desarrollar con éxito su proyecto de vida y alcanzar el perfil profesional, según lo establecido en la currícula.

En todo caso, cobra relevancia dentro del Modelo Educativo de la UEA la metodología de enseñanza y el impacto sobre el aprendizaje estudiantil, en términos de su desempeño. Busca transformar el objetivo educativo de dedicarse principalmente a transmitir conocimientos hacia el reto de desarrollar en el estudiantado la capacidad de auto aprendizaje orientada a "seguir aprendiendo durante toda su vida" y, al mismo tiempo, lograr que sus egresados tengan "empleo para toda la vida", entendida ésta como la capacidad de generar su propio empleo o trabajar en forma independiente (UEA, 2012).

Con respecto a la clasificación de docentes de acuerdo con las estrategias de enseñanza-aprendizaje, se mostraron a los sujetos encuestados las siguientes tipologías y, con base en ello, se les solicitó clasificar al personal docente:

Tradicional: Limita estrictamente su actividad al tiempo educativo, lo más importante es "la trasmisión y fiel interpretación de sus conocimientos", evita intimidad con el alumnado y se mantiene a distancia. Sus clases son aburridas y monótonas, poca promoción de la participación estudiantil, escaso uso de las tecnologías de la comunicación, por sus prejuicios no es capaz de compartir dudas con sus pares de trabajo.

Innovador: Acepta el encuentro con el estudiantado fuera de los ambientes de aprendizaje para optimizar el aprendizaje, se preocupa por este, practica nuevos métodos y técnicas para fortalecer el proceso formativo, promueve la cooperación colectiva con espíritu de cooperación, no es autoritario, pero sabe mantener el orden en el aula, siempre está actualizado, inspira respeto y admiración. Diseña clases dinámicas, permite a sus estudiantes realizar aportaciones, hipótesis sin reprimirlos. Basa su planificación en la prueba diagnóstica.

Mixto: es aquel personal docente capaz de combinar las técnicas tradicionales con las actuales para optimizar el proceso de enseñanza aprendizaje.

En la Figura 1c, se observa que el $54 \%$ de estudiantes considera que el personal docente de la carrera de turismo utiliza una combinación de técnicas tradicionales e innovadoras, mientras que solo un $7 \%$ considera que es netamente innovador. Aunque, la combinación de técnicas puede ser considerada como buena, la idea es el incremento de la creatividad docente durante el proceso formativo.

Con respecto al grado de pertinencia (relación entre: los propósitos institucionales formalmente declarados y los requerimientos sociales), actualización y coherencia de los contenidos en relación con el perfil del estudiantado egresado (existencia de la adecuada correspondencia entre los insumos humanos y físicos, con los procesos y resultados de formación propuestos), el $89 \%$ de sujetos encuestados la considera como buena y solo un $11 \%$ como regular (Fig. 1d).

Sin embargo, cuando se preguntó en qué medida contribuyen los contenidos al logro del perfil profesional, el $57 \%$ consideró medianamente y un $29 \%$ en gran medida. Pareciera que, a pesar de que consideran que los contenidos se ajustan a las asignaturas, no necesariamente estos contribuyen integralmente al logro del perfil profesional del área de turismo.

Es decir, los contenidos deben estar en función de los objetivos de la asignatura, el perfil del estudiantado egresado y los distintos saberes del conocimiento (saber hacer, saber ser, saber convivir y saber conocer) que definen cualquier desempeño académico en los modelos educativos con el enfoque de la complejidad, como es el caso que nos ocupa. Esto saberes integrados en los contenidos curriculares favorecen la creatividad, comprensión y emprendimiento estudiantil, que permite enfrentar con éxito los problemas de su campo laboral (Tobón, 2006 y 2008).

En lo que respecta a en qué medida observó contenidos repetidos entre asignaturas, el 60\% expresó que muy poco y solo un $15 \%$ indicó que medianamente. En este caso, establecer los prerrequisitos y correquisitos 
puede contribuir a evitar la duplicidad de contenidos entre asignaturas, ya que actualmente estos no están considerados en los planes de estudio de la carrera.

En cuanto al balance entre teoría y práctica de los contenidos, el 54\% expresó que era buena y un 36\% que era regular.

Durante la formación de la persona profesional del área de turismo, el balance entre la teoría y la práctica son claves fundamentales en el diseño curricular de los planes de estudios de esta carrera, para garantizar el éxito en el abordaje de los distintos problemas que enfrenta este sector complejo y en constante transformación.

En este sentido, la responsabilidad de la universidad es mantener en el currículo un equilibrio entre los aspectos teóricos y el componente práctico, con la finalidad de fortalecer el desarrollo de habilidades, actitudes y destrezas cognitivas de las futuras generaciones de profesionales. (Paredes e Inciarte, 2006).

Con respecto a la consulta realizada a docentes de la carrera de turismo (Tabla 1) encontramos lo siguiente:

Cuando se les preguntó sobre su participación en la selección de los contenidos, el 57\% respondió haber tenido una participación activa, ya que fueron responsables de elaborar los sílabos de las asignaturas del plan de estudio, desde el Modelo Educativo complejo de la Universidad, que entró en vigencia en el 2012. Así mismo, el $76 \%$ manifestó haber realizado algunos cambios en los contenidos de las asignaturas, con la finalidad de ajustarlos más a las especificidades de la carrera y el perfil del estudiantado egresado.

En este marco, se les consultó si esos cambios habían sido notificados a alguna instancia o habían seguido algunos lineamientos (Fig.2a), el 67\% respondió que no lo habían notificado puesto que desconocían si había que hacerlo y se amparan en el $10 \%$ de ajuste permitido por la Universidad. Mientras que un $27 \%$ afirma haber notificado su acción, pero solo de manera informal.

En este caso, es bueno preguntarse: ¿Los cambios obedecen a actualizaciones o simplemente a la incorporación de contenidos más conocidos por el profesorado? ¿Quién se encarga de supervisar y dar el visto bueno a esos cambios? ¿Son pertinentes y necesarios? ¿Los hacen unilateralmente o por colectivos?
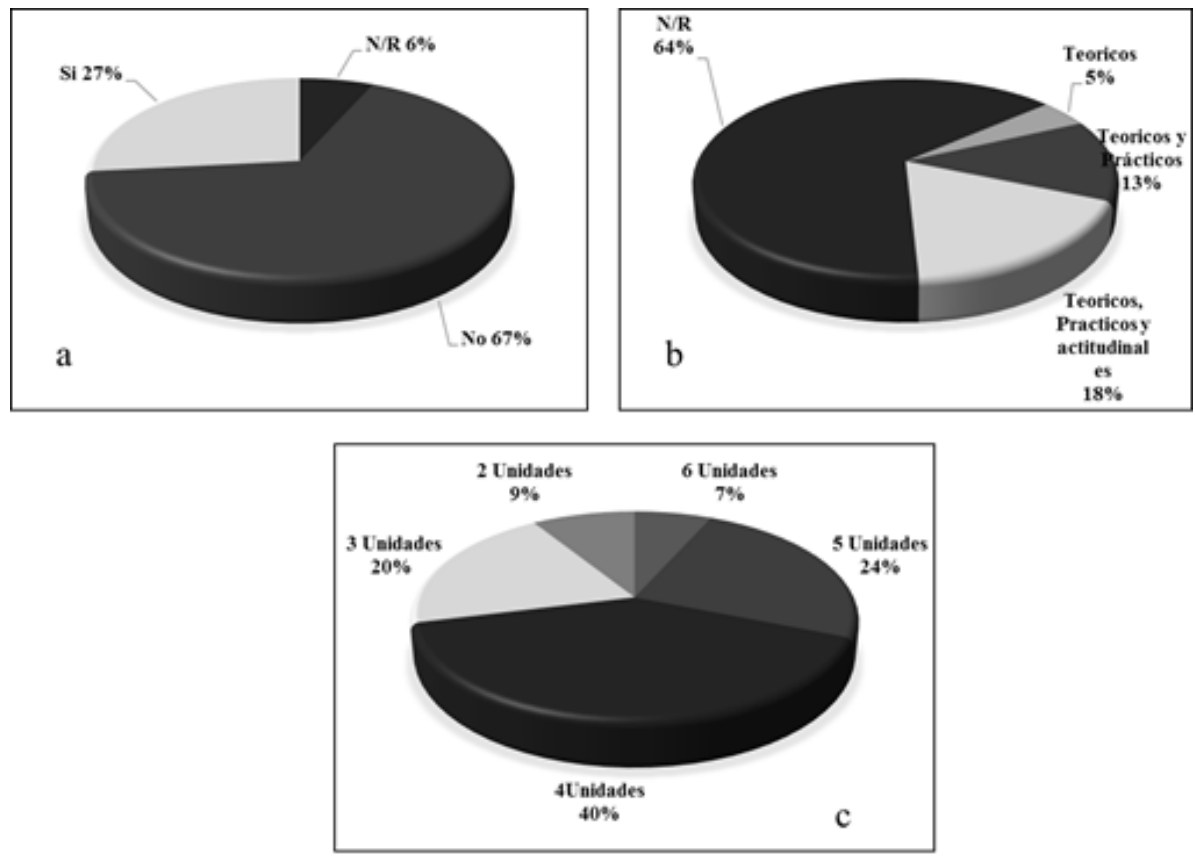

FIGURA 2

2(a) Notificación de cambios en los contenidos; 2(b) Tipos de contenidos y 2(c) Número de unidades didácticas (Fuente: Investigación de campo).

Con respecto al tipo de contenidos presentes en el silabo de cada docente (Fig. 2b), el 64\% no respondió y solo un $18 \%$ emitió una respuesta adecuada, haciendo referencia a contenidos conceptuales, procedimentales 
y actitudinales. Esto es posible, porque el profesorado muestra debilidades en los aspectos didácticos y curriculares. Y esto es corroborado con la solicitud de capacitaciones en estas áreas que hace el personal docente. En este sentido, la Universidad viene haciendo esfuerzos por mejorar estos aspectos, a través de las jornadas curriculares, planificadas al inicio de cada período académico por el Vicerrectorado Académico.

Otro elemento consultado fue el número de unidades didácticas que poseen las asignaturas impartidas por el personal docente encuestado (Fig. 2c). Se observó que la mayor concentración (40\%) se encuentra en asignaturas con cuatro (4) unidades temáticas, seguida de un $25 \%$ con cinco (5) y un $20 \%$ con tres (3). En este apartado llama la atención que un $9 \%$ de docentes declaró tener asignaturas con dos (2) unidades didácticas, y que estas deben ser sometidas a una revisión por parte de especialistas en el área, ya que, a pesar que la tendencia a reducir contenidos poco pertinentes, dos unidades temáticas para impartir durante 16 semanas desde el punto de vista de la planificación académica pareciera poco adecuado, más aún cuando se comparan estos cursos en otras universidades, lo cual puede ocasionar inconvenientes a estudiantes cuando requieren cambio a otra institución.

En este mismo orden, cuando se preguntó, si consideraban que el número de unidades didácticas de su asignatura era suficiente para alcanzar los resultados de aprendizaje del perfil y los objetivos de la asignatura, solo un $18 \%$ lo consideró insuficiente, mientras que el (82\%) planteó que se ajusta a las necesidades y perfil profesional de la carrera. En todo caso, se estima conveniente reflexionar sobre este aspecto con docentes y especialistas del área y revisar sílabos de otras universidades, tanto nacionales como internacionales, para plantear propuestas adecuadas a las necesidades y demandas de la sociedad, tomando en cuenta el nuevo Reglamento del Régimen Académico que busca favorecer la movilidad de estudiantes y la transferencia de créditos.

El uso de las estrategias de enseñanza-aprendizaje empleadas por docentes para el logro de los resultados de aprendizaje fueron diversas y mostraron la siguiente tendencia: Un $80 \%$ aplica las conferencias magistrales, un $10 \%$ los debates, foros, talleres, trabajo en grupo, $7 \%$ el uso de estudio de casos, resolución de problemas, prácticas de campos y de laboratorio y tan solo un 3\% manifestó incluir los organizadores gráficos (mapas mentales y conceptuales) como estrategias. En cuanto a los instrumentos de evaluación, no parece haber tanta claridad en este aspecto, la totalidad de los sujetos encuestados confunde instrumentos de evaluación (rúbricas, lista de cotejo, escala de valoración, entre otros) con las estrategias para verificar el logro de los resultados de aprendizajes.

Estos datos llaman a la reflexión, ya que el alcance de los resultados del aprendizaje de estudiantes, pasa por tener claridad sobre los criterios e instrumentos de su evaluación.

Por tanto, los resultados del aprendizaje deben escribirse de tal forma que permitan ser observados o monitoreados en la medida de lo posible, ya sea directa o indirectamente. En todo caso, preguntas como: ¿cómo demuestra el estudiantado lo que ha aprendido?, ¿cómo manifestará que ha alcanzado un resultado del aprendizaje concreto?, ¿cómo se puede observar que un resultado del aprendizaje definido ha sido adquirido? pueden ayudar a reflexionar sobre los métodos e instrumentos de evaluación más adecuados para que cada estudiante evidencie su aprendizaje. Los procedimientos de evaluación deben, por tanto, diseñarse minuciosamente, proporcionarse a estudiantes y revisarse de manera periódica (ANECA, 2013, citado por Herrero et. al., 2014).

En cuanto a las sugerencias para la actualización de los contenidos propuestas por el personal docente encuestado (Figura 3), pueden ser resumidas en cuatro (4) categorías: un 51\% estima conveniente que las actualizaciones se realicen formando equipos de trabajo con especialistas de las disciplinas y docentes de las asignaturas, un 33\% expresa la necesidad de revisar los contenidos de las asignaturas dictadas en universidades nacionales e internacionales, un $11 \%$ plantea que a través de la participación en proyectos de investigación de su área de conocimiento, cuyos resultados pueden ser tomados en cuenta a la hora de realizar una actualización y un 5\%, correspondiente con profesorado de menor experiencia en la asignatura que está impartiendo, expresó que es necesario recibir capacitación. 
En todo caso, los resultados muestran una tendencia que apunta a un trabajo en equipo, no dejar de lado la investigación, el perfil del estudiantado egresado, objetivos de la asignatura, el Modelo Educativo de la Universidad, los adelantos tecnológicos y las tendencias mundiales en temas de interés para cada asignatura, que deben ser monitoreados por las autoridades pertinentes, entre otras cosas.

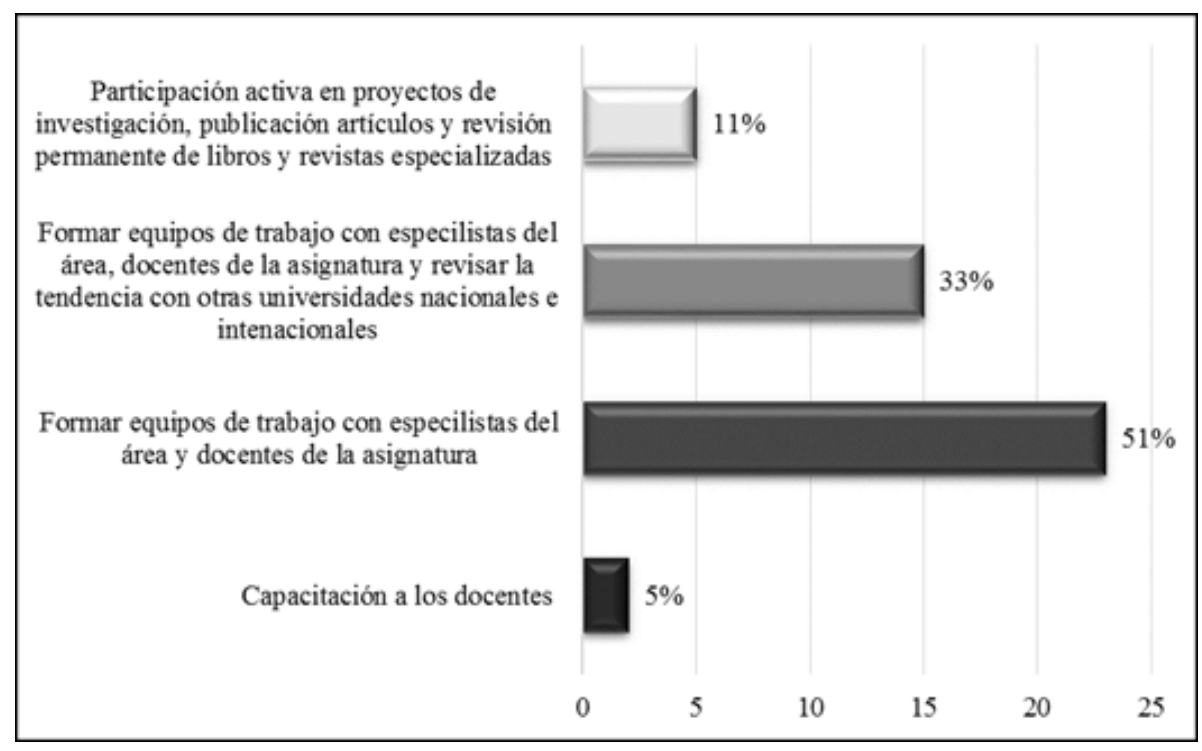

FIGURA 3

Sugerencias para actualización de contenidos del sílabo. (Fuente: Investigación de campo).

Finalmente, para completar la valoración interna sobre el objeto de estudio, se recibió la opinión de las principales autoridades académicas de la Universidad Estatal Amazónica, por medio de la entrevista personalizada.

En líneas generales, las autoridades entrevistadas (Vicerrectora, Decana y Coordinadora de la carrera de turismo) están conscientes de que, a pesar de que la Universidad cuenta con un modelo educativo adaptado a las tendencias curriculares actuales, hay una brecha entre lo escrito y la puesta en práctica del modelo por parte de los docentes (instrumentación curricular). En este sentido, la Universidad viene haciendo esfuerzos desde el Vicerrectorado Académico, para buscar mecanismo que permitan mejorar y hacer más eficiente el proceso de enseñanza-aprendizaje. Para ello, se planifica la semana curricular y jornadas de reflexión al inicio de los períodos académicos, talleres y capacitaciones en el área didáctica.

Sin embargo, estas acciones tienen que ser sistemáticas y estar sometidas a un seguimiento y valoración de su impacto en la calidad del estudiantado egresado.

$\mathrm{Al}$ respecto, vale la pena acotar lo siguiente: La etapa de instrumentación curricular corresponde a la preparación que hará posible la aplicación del currículo, para contar con el menor número de improvisaciones en cuanto a recursos, procedimientos y desempeño docente. Los elementos que conforman esta etapa son:

Formación docente: se refiere a la mejora del quehacer docente, es decir, capacitar al personal académico en las tareas específicas que debe desempeñar cuando asume el compromiso de guiar el proceso de enseñanzaaprendizaje. Para ello, es necesario implementar un plan de desarrollo profesional docente, a través del acompañamiento pedagógico permanente.

Planeamiento didáctico: corresponde a la planificación del proceso de enseñanza aprendizaje, que cada docente debe realizar, con el fin de contar con todo lo necesario para lograr el aprendizaje esperado. Se sugiere la realización de jornadas de reflexión o socialización que le oriente en la selección de las metodologías de enseñanzas, el sistema de evaluación y la actualización de los contenidos didácticos. 
Recursos: hace referencia a las herramientas didácticas necesarias para llevar a cabo el proceso de enseñanza aprendizaje, ya sean guías, ejercicios, casos, etc., los cuales deben ser coherentes con el perfil de egreso, los objetivos de la carrera y de la asignatura.

Por otra parte, en cuanto al tema de la actualización de los planes de estudios, unánimemente estimaron conveniente revisar este aspecto, ya que estos, al igual que los conocimientos, no son estáticos y sería pertinente contar con una guía orientadora.

En este sentido, cuando se les preguntaron sus sugerencias para realizar el proceso de actualización curricular de los sílabos, la totalidad coincide en que hay que considerar los objetivos de la carrera, el perfil profesional, los resultados de aprendizaje en cada nivel donde se desarrolla la asignatura, el contexto, la disciplina a la que pertenece, los avances de la ciencia, la aplicación de las soluciones según las necesidades del entorno, y los estudios metodológicos de los colectivos docentes desarrollados en semestres anteriores y, sobre todo, el trabajo en equipos multidisciplinarios.

Todos los aspectos señalados anteriormente son de vital importancia para mejorar la calidad de la enseñanza, pero el éxito va a depender del seguimiento que se haga a dicho proceso, de tal manera que permita conocer cómo está resultando, qué debilidades y fortalezas se están presentando en la nueva propuesta y así poder realizar los ajustes micro curriculares en la organización y estructuración del plan de formación curricular, como también las mejoras de manera oportuna.

\section{Evaluación externa}

Esta evaluación busca, por una parte, conocer el impacto que ha tenido una determinada carrera en el medio externo y, por otra, recoger las nuevas necesidades, tendencias y requerimientos de la profesión (Del Castillo,G., 2004; Louro et al, 2011), considerando:

Impacto social: Permite conocer el impacto que han tenido los grupos profesionales de la carrera en sus diversos ámbitos de desempeño, sus funciones, intervención en las necesidades y problemas sociales, etc. Esta información se recoge a través de personas y fuentes externas al estudiantado egresado y a la universidad. En nuestro caso, entrevistamos algunos entes empleadores en el área de turismo (Tabla 2).

Estudiantado graduado: Es necesario realizar el seguimiento del estudiantado egresado de la carrera objeto de estudio, conocer su ocupación, éxito, empleabilidad, tiempo que demoró en emplearse, valoración de los contenidos de las asignaturas, su pertinencia y apoyo en su desempeño profesional, entre otros indicadores que permiten dimensionar los resultados del proceso formativo.

En la Tabla 3 se discriminan, por género, los indicadores más relevantes, obtenidos a través de la aplicación de las encuestas respectivas.

TABLA 3

Distribución de los indicadores por género

\begin{tabular}{llll}
\hline Indicador & Total & Varones & Mujeres \\
\hline Intención de estudios de cuarto nivel & 4 & 2 & 2 \\
Nivel de empleo & 14 & 5 & 9 \\
Satisfacción por los conocimientos adquiridos & 14 & 5 & 9 \\
Sector empleador- público & 12 & 4 & 8 \\
Sector empleador-privado & 2 & 2 & - \\
\hline Total entrevistado & 20 & 7 & 13
\end{tabular}

Nota: Elaboración propia 
Estos resultados indican una mayor presencia del género femenino en la carrera de turismo (65\%), lo cual se corresponde con lo expresado en el Informe Mundial sobre las Mujeres en el Turismo 2010 (Organización Mundial de Turismo, 2013) donde se destaca que aproximadamente el $72 \%$ de estudiantes de tercer nivel en el área de servicios en el mundo son mujeres, en contraste con el 38\% de profesoras de tercer nivel en la misma área. Esta distribución se refleja en los indicadores analizados.

Con respecto a la pregunta sobre si siguen estudios de cuarto nivel (Fig. 4a y Tabla 3), solo el 20\% expresó estar realizando estudios de maestrías en áreas algo relacionadas con su formación, así mismo señalaron la necesidad de que la universidad brinde esa posibilidad, ya que tienen que trasladarse a otras provincias lo que hace más elevado los costos de estos estudios.
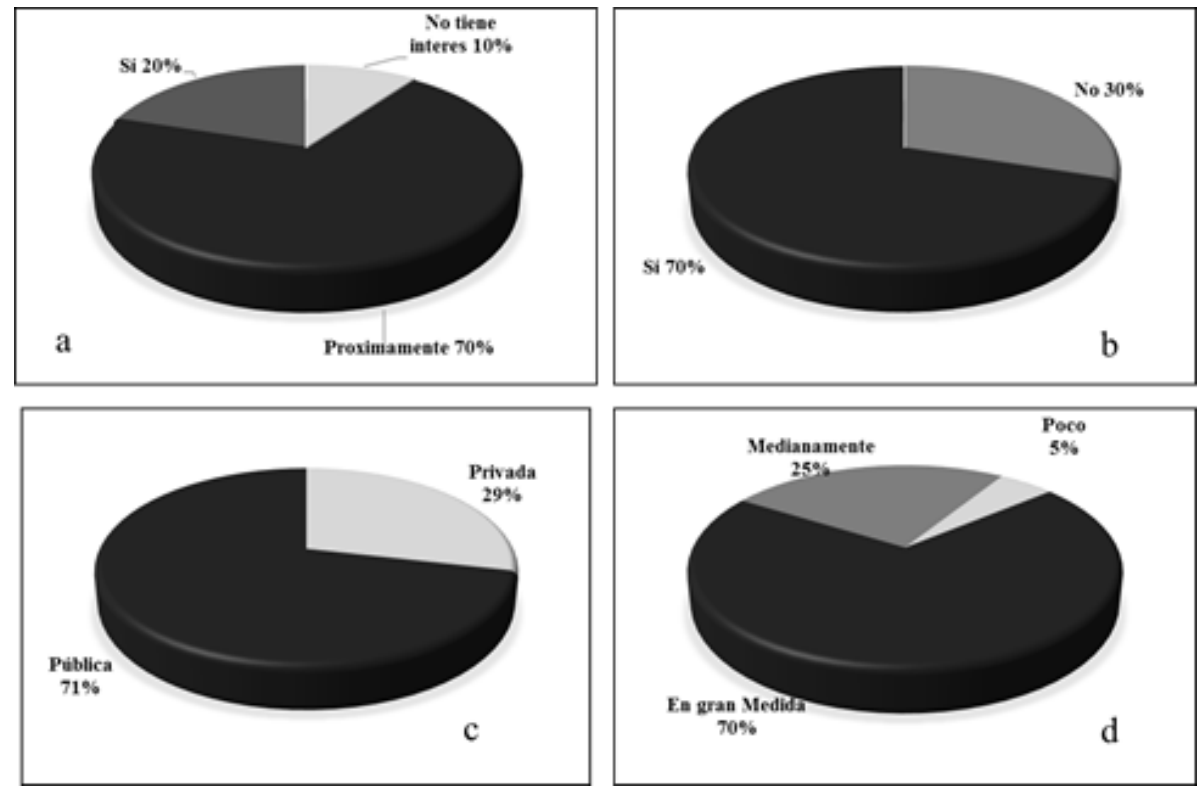

FIGURA 4

4(a) Intención de continuar estudios de cuarto nivel; 4(b) Nivel de empleo; 4(c) Tipo de empresa y 4(d) Satisfacción por los conocimientos adquiridos. Fuente (Investigación de Campo).

Por otro lado, un alto porcentaje (70\%) expresó su interés de continuar con sus estudios de cuarto nivel, ya que lo consideran necesario por los niveles de competitividad en esta área, a la hora de buscar puestos de trabajo. Sin embargo, limitaciones de tiempo, dinero y el no contar en la zona con un posgrado en su área de formación hacen que esta aspiración no se concrete.

En este contexto, se pone de relieve la importancia de los estudios de posgrados para el desarrollo de las competencias, la superación profesional y el fortalecimiento de la capacidad de aprendizaje para toda la vida (Manzo, et. al, 2006). También es una oportunidad para la Universidad Estatal Amazónica para ampliar su oferta académica y contribuir con el desarrollo científico de la región.

En cuanto a la pregunta si actualmente están trabajando (Fig. 4b y Tabla 3), un alto porcentaje (70\%) manifestó contar con un empleo, de esta población el 75\% encontró un puesto de trabajo antes del año, mientras que el $25 \%$ restante tardo más de un año para empelarse. Estos resultados son alentadores tanto para estudiantes como para la universidad, pues manifiestan un alto grado de ocupación.

Respecto al tipo de institución donde presta sus servicios (Fig. 4c y Tabla 3), el 71\% manifestó estar trabajando en empresas del sector público, principalmente gobiernos provinciales, parroquiales, municipales y Ministerio de Educación, en actividades técnicas o relacionadas con su área de formación. Mientras que solo un 29\% se encuentra laborando en el sector privado (empresas de servicios turísticos, ONG).

De estos resultados se podría inferir que, a pesar que la carrera de turismo dictada en la UEA se desarrolla en el cantón Pastaza, provincia Pastaza, situada en la Región Amazónica, caracterizada por la abundancia de 
recursos turísticos naturales que hacen de esta actividad un área estratégica para el desarrollo económico de la provincia, por su potencial de generación de ingresos y empleo, y por sus encadenamientos intersectoriales que incentivan inversiones en otros sectores, el nivel de empleabilidad de profesionales de esta área es menor que la privada. Esto quizá pueda tener su explicación, por una parte, en la contratación de personal técnico no necesariamente formado en las universidades y la falta de consolidación de este sector en la provincia, lo que puede verse como una oportunidad para profesionales que se han graduado en esta institución, de incursionar en esta área como gestores de su propia empresa (ejercicio libre de la profesión).

En la Figura 4d y Tabla 3, se puede observar en qué medida los grupos profesionales graduados valoran los conocimientos adquiridos en la UEA durante su proceso de formación para el ejercicio de su profesión.

En cuanto a ello, el $70 \%$ consideró de gran utilidad los conocimientos adquiridos, donde el aprendizaje permanente es un valor inherente a su profesión.

Sin embargo, este porcentaje disminuye cuando se les preguntó en qué medida los contenidos del plan de estudio se adaptaban a las necesidades del entorno laboral. Un 57\% los consideró pertinentes y contextualizados, mientras que un $5 \%$ consideró que no se ajustaban a las demandas actuales del campo laboral. Estos resultados, aunque pueden ser valorados como positivos globalmente, no deben perderse de vista, por cuanto ese $5 \%$ expresó que los contenidos vistos durante su formación no son los más pertinentes y contextualizados.

Con respecto a la pregunta si habían observado repetición de contenidos entre asignaturas del plan de estudio, el $65 \%$ expresó que no, mientras que un $25 \%$ consideró que un poco y un $10 \%$ que medianamente. Estas cifras pueden servir como un llamado de atención, si bien es cierto que la repetición de contenidos es baja, es necesario estar alerta a esta situación y aplicar los correctivos necesarios.

En la Figura 5, se observan las tres categorías que resultaron al preguntar sobre las capacidades que deben ser fortalecidas durante el proceso de formación del estudiantado graduado en turismo para alcanzar el éxito en su desempeño profesional. El 60\% expresó que el liderazgo, emprendimiento y el desarrollo de nuevos productos son áreas que deben ser potenciadas en los planes de estudios, seguida de la evaluación de proyectos y manejo de herramientas informáticas especializadas (52\%) y, finalmente, el dominio de un segundo idioma y creatividad en la solución de problemas con un $15 \%$.

En este sentido, más del $80 \%$ consideró necesario que estas capacidades deben ser potenciadas desde todas las asignaturas que conforman el plan de estudio, ya que forman parte fundamental del perfil de egreso.

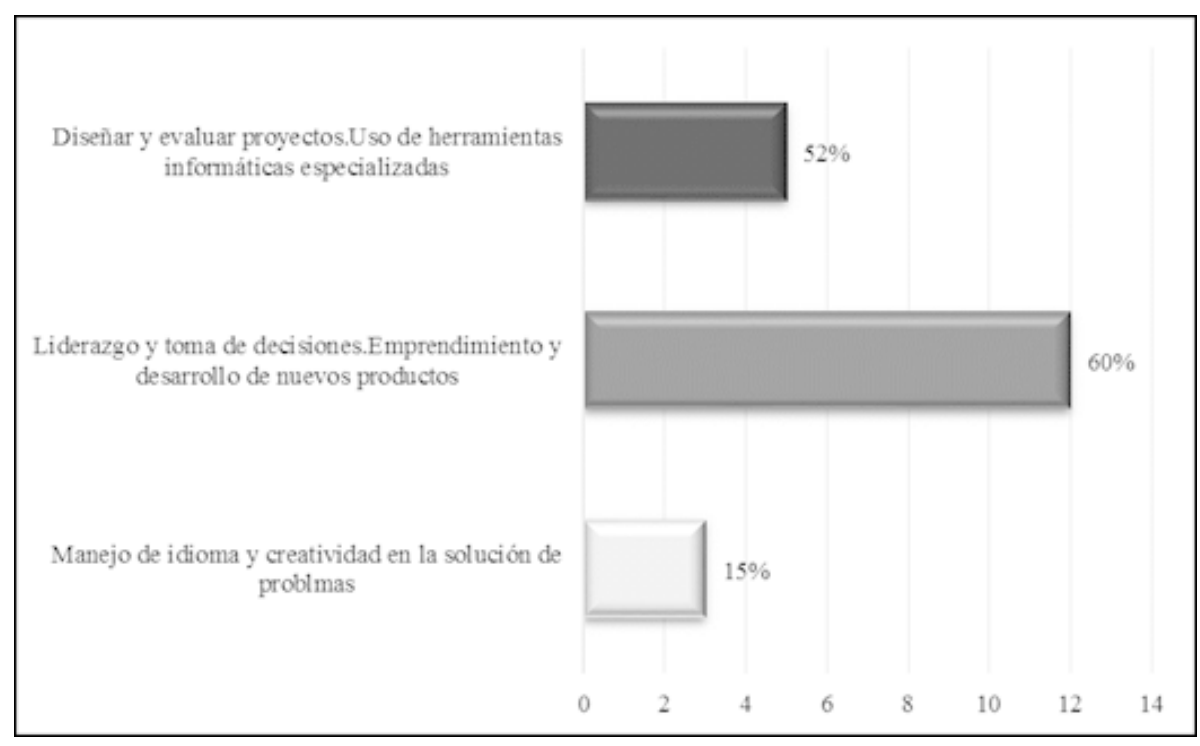

FIGURA 5

Capacidades que se deben fortalecer. Fuente (Investigación de campo) 
Con respecto a las principales competencias que deben mostrar profesionales que contratan, el $75 \%$ expresó como fundamentales: liderazgo, uso efectivo de las TIC, dominio de un segundo idioma; mientras que el 25\% restante planteó: trabajo en equipo, evaluación de proyectos y uso efectivo de la comunicación oral y escrita.

El 79\% de las instituciones empleadoras encuestadas (Tabla 2) valoraron como buenas las capacidades mostradas en el ejercicio profesional por los profesionales que se han graduado y un $21 \%$ como excelentes.

Cuando se les solicitó la opinión en cuanto a los valores mostrados por estos grupos de profesionales de la UEA (honestidad, responsabilidad, ética, compromiso, entre otros), el 90\% expresó que eran muy buenos, y el $100 \%$ que contrataría a profesionales provenientes de la Universidad Estatal Amazónica en el área de turismo, siempre que exista una demanda insatisfecha.

\section{CONCLUSIONES Y RECOMENDACIONES}

La metodología propuesta para realizar la evaluación curricular de la carrera de turismo a través de una evaluación interna y externa permitió identificar lo expuesto por Roldan (2004, p.11): “los aciertos, las fallas, las debilidades y los ajustes necesarios que se requieren para ponerlo acorde con el desarrollo científico y tecnológico y con las demandas de la sociedad a la que servirá el profesional que se forme con ese plan de estudio".

La aplicación de esta metodología a todas las carreras vigentes de la UEA, puede ser un aporte para favorecer la cultura de la evaluación y contribuir a los procesos dinámicos y continuos de acreditación institucional. En todo caso, una institución de educación superior cuyos planes de estudio estén interconectados al fortalecimiento social y al desarrollo de la ciencia y tecnología, como es el caso de la Universidad Estatal Amazónica, está obligada a mantener planes de estudios actualizados y contextualizados.

La evaluación interna permitió detectar las debilidades docentes en el manejo de los componentes curriculares, diferencias en los contenidos de una misma asignatura cuando son dictados por profesorado distinto, unidades curriculares con un número bajo de unidades didácticas y la falta de un mecanismo que oriente a docentes en la actualización de los contenidos curriculares de los sílabos que conforman el plan de estudio de la carrera. Por su parte, el estudiantado percibe al personal docente con buena preparación, con poca repetición de contenidos de un curso a otro, que aplica de manera combinada estrategias de enseñanzas tanto innovadoras como tradicionales y con contenidos contextualizados y relacionados con el perfil del egresado.

Los resultados de la evaluación externa de la carrera de turismo denotan que integralmente el estudiantado graduado está satisfecho con su formación y los entes empleadores con las competencias demostradas por profesionales de la UEA.

Sin embargo, los resultados muestran que un 30\% del estudiantado graduado que se entrevistó no está satisfecho con los conocimientos adquiridos y un $5 \%$ los consideran poco pertinentes y contextualizados. Esto sugiere la necesidad de complementar y mejorar la calidad académica de los planes de estudios, tomando como referencia las necesidades locales y nacionales de la industria turística.

Para mejorar las competencias del estudiantado egresado, la Universidad Estatal Amazónica cuenta actualmente con una Maestría en Gerencia Turística. Su objetivo es formar a profesionales del turismo capaces de liderar, adoptar y emprender procesos de innovación y de cambio en este sector y de dar respuesta a las necesidades de las organizaciones y de la sociedad actual.

Así mismo, para favorecer su inserción laboral, la UEA ha implementado mecanismos para aumentar el número de convenios con instituciones y empresas tanto del sector público como privado del área turística.

Una de las limitaciones de esta investigación fue la dificultad para localizar a un mayor número de personas graduadas, debido a la debilidad en la política de seguimiento, monitoreo y actualización de la base de datos de este componente. 
Finalmente se recomienda incorporar una línea de investigación en el área de planificación y evaluación curricular, como un proceso de investigación colaborativa e inherente al ejercicio de la docencia, que permita valorar y realizar seguimiento del impacto de los planes de estudios de las carreras dictadas en la UEA en la calidad de la enseñanza de su estudiantado egresado.

\section{Agradecimientos}

Las personas autoras agradecen al Programa Ateneo de la SENESCYT-Ecuador y a la Universidad Estatal Amazónica (UEA), por todo su apoyo logístico y económico que hizo posible el trabajo de campo.

\section{REFERENCIAS}

Briceño, M. y Chacín, M. (2008). Elementos teóricos para sustentar la evaluación curricular como proceso de transformación y construcción social de las universidades venezolanas. Investigación y Postgrado, 23 (3), 69-87. Recuperado de http://www.scielo.org.ve/scielo.php? script=sci_arttext\&pid=S1316-00872008000300004\&lng=es\&tlng=pt.

Del Castillo, G. (2004). El impacto de la evaluación externa en dos instituciones de educación superior en México. Revista Perfiles Latinoamericanos, 12(25), 115-148.

Díaz Barriga, Á. (2005). El profesor de educación superior frente a las demandas de los nuevos debates educativos. Perfiles educativos, 27(108), 9-30. Recuperado de http://www.scielo.org.mx/scielo.php? script=sci_arttext\&pid=S0185-26982005000100002\&lng=es\&tlng=es.

Fonseca Casciol, N., y Pereira de Homes, L. (2011). La evaluación curricular en la Universidad del Zulia: Casos Facultades de Odontología y Ciencias Económicas y Sociales. Paradigma, 32(1), 7-31. Recuperado de http:// www.scielo.org.ve/scielo.php?script=sci_arttext\&pid=S1011-22512011000100002\&lng=es\&tlng=es.

García Sanz, M., García Sánchez, F., Martínez Segura, M. y Maquilón Sánchez, J. (2013). Diseño, aplicación y evaluación de un programa para mejorar la formación evaluativa del profesorado de educación especial. Educatio Siglo XXI, 31(1), 149-172.

Herrero, R., Ferrer, M. y Calderón, A. (2014). Evaluación de las competencias genéricas mediante rúbricas. Recuperado de http://repositorio.upct.es/bitstream/handle/10317/4115/ecg.pdf?sequence=1\&isAllowed=y

Inciarte G., A. y Canquiz R., L. (2009). Metodología para el diseño de perfiles basados en el enfoque de competencias. Laurus, 15(29), 33-52. Recuperado de http://www.redalyc.org/articulo.oa?id=76120642003

Inciarte González, A. y Canquiz Rincón, L. (2001). Análisis de la consistencia interna del currículo. Informe de investigaciones educativas, 15(1-2), 79-90.

Inciarte, A. (1996). Un modelo para el desarrollo del currículo de la educación superior. Revista Agenda Académica, 3(2). Caracas: UCV-Sadpro.

Louro Bernal, I., Perdomo, I., Gálvez González, A. y Sanabria Ramos, G. (2011). Estrategia de preparación para la evaluación externa de maestrías. Educación Médica Superior, 25(2), 107-115.

Manzo Rodríguez, L., Rivera Michelena, C. y Rodríguez Orozco, A. (2006). La educación de posgrado y su repercusión en la formación del profesional iberoamericano. Educación Médica Superior, 20(3) Recuperado de http:// scielo.sld.cu/scielo.php?script=sci_arttext\&pid=S0864-21412006000300009\&lng=es\&tlng=pt.

Marín, H (2015). Propuesta metodológica para la actualización curricular de syllabus de las carreras dictadas en la UEA. Proyecto financiado por la SENESCYT-Ecuador, a través del programa Ateneo (Inédito). Universidad Estatal Amazónica, Puyo-Ecuador.

Mata, Y. (2010). Pertinencia del ajuste curricular de la carrera de educación mención preescolar de la Universidad Nacional Abierta (Tesis de maestría). UCV. Caracas, Venezuela.

Mateo, J. (2006). Claves para el diseño de un nuevo marco conceptual para la medición y evaluación educativas. Revista de Investigación Educativa, 24(1), 165-186. 
Organización Mundial Del Turismo (OMT). (2013) Informe mundial sobre las mujeres en el turismo 2010. Madrid. Recuperado de http://www.e-unwto.org/doi/pdf/10.18111/9789284414789.

Ortiz, M. y Borjas, B. (2008). La investigación acción participativa: Aporte de Fals Borda a la educación popular. Espacio Abierto, 17(4), 615-627.

Paredes, I. e Inciarte, A. (2006). Relación teoría-práctica en el quehacer curricular de la mención Educación Básica Integral. Revista Omnia, 12(2), 124-147. Recuperado de http://www.redalyc.org/articulo.oa?id=73712206

Pérez, R. (2007). La evaluación externa y sus implicaciones: Aspectos técnicos, prácticos y éticos. Avances en supervisión educativa: Revista de la Asociación de Inspectores de Educación de España, 6(7), 1-23. Recuperado de https://avances.adide.org/index.php/ase/article/view/275/235

Roldán, L. (2004). Elementos para evaluar planes de estudios en educación superior. Revista Educación, 29 (1), 11-123. Costa Rica.

Salas Perea, R. (2016). Is curriculum redesigning without previous curricular assessment scientific? Educación Médica Superior, 30(2). http://scielo.sld.cu/scielo.php? script=sci_arttext\&pid=S0864-21412016000200017\&lng=es\&tlng=en

Salas, R. y Salas M. (2014). Evaluación curricular. En Educación médica contemporánea. Retos, procesos y metodologías. Bucaramanga: Imprenta Universidad de Santander-UDES.

Sánchez González, María. (2010). Técnicas docentes y sistemas de evaluación en educación superior. Madrid, España: Ediciones Narcea S.A.

Tobón, S. (2006). El diseño del plan docente en información y documentación acorde con el espacio europeo de educación superior. Madrid: Editorial Universidad Complutense de Madrid.

Tobón, S. (2008). Gestión curricular y ciclos propedéuticos. Bogotá: ECOE.

Universidad Estatal Amazónica. (2012). Modelo complejo por resultados de aprendizajes. Pastaza-Ecuador: Autor.

\section{BY-NC-ND}

